\title{
CYBERCROMLECH: A NEW FRAMEWORK FOR COLLECTIVE BEHAVIOUR GAME EXPERIMENTS
}

\author{
Alexey Botchkaryov \\ Department of Computer Engineering \\ Lviv Polytechnic National University \\ Bandery Str., 12, 79035, Lviv, Ukraine \\ E-mail: alb@polynet.lviv.ua
}

\author{
Serhiy Kovela, \\ School of Business Information \\ Management, Kingston University \\ Kingston Hill, Kingston, KT2 7LB, UK \\ E-mail: S.Kovela@kingston.ac.uk
}

\section{KEYWORDS}

CyberCromlech, multiagent systems, collective behaviour, game experiments.

\begin{abstract}
A new approach to modelling multiagent interactions using the CyberCromlech framework is proposed in the paper. The main distinction of the approach is the capability to flexibly assess and control the dynamic structure of cause-effect connections emerging from a common history of multiagent interactions. Based on this capability, we have designed several game experiments to study various aspects of collective behaviour, with the most interesting of those being discussed in the paper.
\end{abstract}

\section{INTRODUCTION}

Research in the field of multiagent systems (Woolridge 2002; Weiss 2000) gains ever increasing importance in view of technological breakthroughs in design of wireless communications and computing facilities. New possibilities require new approaches to the design of information technologies, which stipulates the need for intensive research in such fields as classical AI, game theory and machine learning, etc. Characteristic example of such research is the design of test problems or task environments. Here the goal is to create and study artificial rational agents capable of solving problems set in those task environments. Examples of such test problems are robotic soccer (including the virtual soccer), the tower building problem (Ishida 1997), the tile world problem (Pollack and Ringuette 1990), the mechanical balancing problem (Botchkaryov 2002) and many others.

The paper proposes a new approach to the design of test problems (called further "game experiments") based on the CyberCromlech framework. The approach is based on the idea of storing and utilizing the history of agent interactions in a form that is explicit and convenient enough to allow for study of spontaneous emergence of collective behaviour. To demonstrate the idea, formulations of several game experiments using the CyberCromlech framework are presented with one of them - the temporizing game - being examined in more detail. In conclusion, we give the vision of further evolution and potential practical application of the approach proposed.

\section{CYBERCROMLECH}

\subsection{Objectives}

Analysis of the existing test problems in the field of multiagent systems was used in the study. This allowed us to formulate the set of requirements to the new approach, most important of those being: 1) the capability to take stock of and control the dynamic structure of cause-effect connections, emerging from interactions between agents, with a great deal of flexibility; 2) the capability to flexibly control the level of uncertainty (lack of information about environment) and freedom of actions for every interacting agent; 3) a sufficient level of abstraction, allowing for a wide range of interpretations of processes under study.

\subsection{Definitions}

\subsubsection{CyberCromlech Definition}

CyberCromlech is: 1) a spatial structure, used to host agents in its nodes; 2) discrete timing, used to synchronize agent interactions; 3) rules defined on the structure and timing to govern: a) the way agents traverse the structure; b) the way the structure reacts to the traversal by changing node states.

\subsubsection{Spatial Structure}

A basic version of the spatial structure is analogous to a generalized plan of megalithic constructions cromlechs (Fig.1), is named after them accordingly. The CyberCromlech structure (Fig.2) is specified by L layers (concentric circles) and $\mathrm{R}$ rays (connecting outer and inner circles). Intersections of layers with rays produce a network of interconnected nodes through which agents can pass. Agents may only stop in nodes and move between them through existing transitions. In the very middle of cromlech there is the inner area, outside of it there is the outer world - any agents' moves in either of those have to be specified additionally.

\subsubsection{Discrete Timing}

Movement of agents (i.e. transitions from one node to another) is timed discretely using a common discrete clock. That is, time is counted by steps and all moves that occur in the same step are considered simultaneous. 


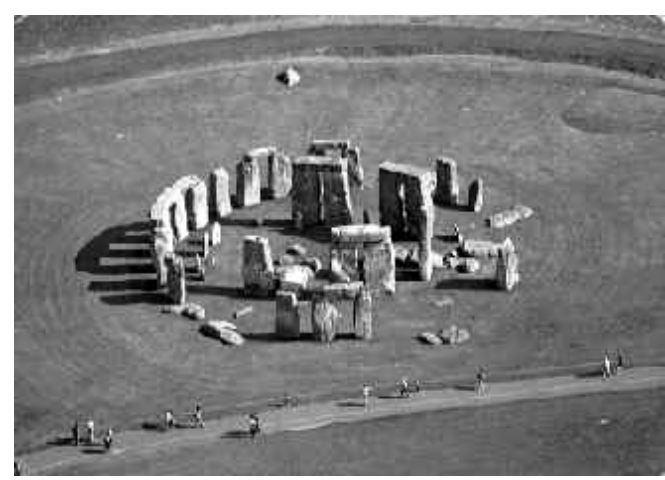

Figure 1. CyberCromlech prototype - Stonehenge

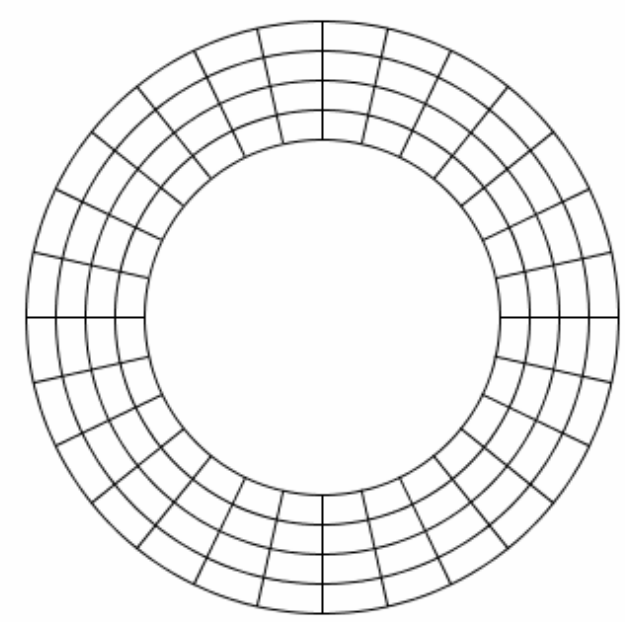

Figure 2. CyberCromlech spatial structure $(L=5, R=28)$

\subsubsection{Movement Rules}

There are the following rules governing movement of agents in CyberCromlech. 1) There can be only one agent in each single CyberCromlech node at a time. 2) In every step, an agent has to choose one of the "free" adjacent nodes and move into it. Special cases (exceptions) where such transition is not possible are specified additionally. 3) An agent cannot go back to the node it left on the previous step, i.e. only progressive movement is allowed.

\subsubsection{Rules for Changing Node States}

Agent transitions between CyberCromlech nodes change those nodes' states in the following way.

1) Every node can be in one of two states: "open” or "closed". If a node is "open”, an agent can move into it, otherwise it cannot. 2) By passing through a node, an agent changes its state to "closed" for a limited number of steps (the corresponding counter for this node is set to $C_{0}$ ). 3) Every next step, the counter is decreased by 1 . The node's state is reset back to "open" when the counter reaches a 0 value again.

The above basic set of rules can be modified to allow agents greater freedom of movement between nodes.
This can be achieved by allowing transition trails of different agents to intersect. Below follows the modified set of rules ("Celtic knot" rules): 1) A node can be in one of three states: "open", "semi-open", "closed". 2) By passing through a node and maintaining direction of movement (i.e. moving along level or ray without turning) an agent changes this node's state to "semiopen". 3) By passing through a node and changing direction of movement, an agent changes this node's state to "closed". 4) By entering a "semi-open" node (only possible if moving perpendicularly to the existing trail passing the node), an agent changes this node's state to "closed".

\subsection{Explanations}

The definitions given require some additional explanation. 1) On every step an agent must choose between the nodes available and decide where to move next. Moving in such a manner, the agent is performing a sequence of elementary choices. 2) While traversing CyberCromlech, every agent is forming a $\mathrm{C}_{0}$-long trail of "closed" (or "closed" and "semi-closed" for "Celtic knot" rules) nodes (Fig.3,4). 3) Rules of some games may stipulate: a) a limited or unlimited $\left(\mathrm{C}_{0}=\infty\right)$ trail length; b) different trail lengths for different agents; c) variable trail lengths. 4) Sets of "open" nodes in CyberCromlech are dynamically changing as the game evolves quite analogously to the maze with a dynamically changing structure. The peculiarity here, however, is in the fact that it is the agents that move inside the maze that are changing its structure. 5) Agents' interactions span over time as they cross each other's trails. In this regard crossing one's trail by another agent can be interpreted in special way.

\subsection{Conformance to requirements}

Let us examine if the approach proposed conforms to formulated requirements.

1. In the proposed approach, the emerging structure of cause-effect connections is modelled by a dynamic system of trails formed by agents traversing CyberCromlech. Agents' actions become the reason for general limitations affecting all their future actions. By altering length and, possibly, some other trail characteristics one can take stock of and control causeeffect connections emerging in agent interaction.

2. The level of information distribution about the overall situation is controlled by an individual level of uncertainty. That is, the more nodes an agent can see around, the more it is informed. Freedom of movement for agents can be controlled by altering the maximal number of nodes it can pass in a single step.

3. The large number of interpretations of elementary choices that agents make on every step gives broad possibilities for interpretation of agent interactions. That is, depending on what the developer perceives 
CyberCromlech nodes to be, there could be different variants of interpretation of modelled agent interaction process.

\section{GAME EXPERIMENTS}

\subsection{Objectives}

It is possible to study various aspects of collective (multi-agent) behaviour in CyberCromlech using game experiments, where agents play a given game. It is necessary here for the developer to be able to adequately reflect the collective behaviour problem in objectives and rules of the game. On the other hand, one could try identifying and studying specific aspects of collective behaviour in independently developed game. To demonstrate the approach proposed, four examples of games utilizing CyberCromlech (twine game, pursuit-escape game, occupancy game, temporizing game) are presented. All the games under study imply that a player can only see the state of adjacent nodes and can only make one transition between nodes in a single step. A node's states change according to "Celtic knot" rules. It is also implied that players do not know the total number of players (N) and CyberCromlech dimensions (L, R).

\subsection{Game examples}

\subsubsection{Twine game}

Twine game is one of the simplest games modelled in the CyberCromlech. Agents are arbitrarily positioned in CyberCromlech nodes at start of the game. While traversing CyberCromlech, agents form trails of "closed" and "semi-closed" nodes, moving through one node at each step. If an agent cannot make a move due to all adjacent nodes being "closed", it is "trapped" and loses the game. The winner is the agent being trapped last (there could be several of them). The game is guaranteed to finish in finite number of steps provided the trail length is unlimited $\left(\mathrm{C}_{0}=\infty\right)$. On average, the lesser the number of agents $(\mathrm{N})$ to the number of nodes (M) (i.e. the more free space there is in CyberCromlech) is, the longer the game will continue.

\subsubsection{Pursuit-escape game}

It is proposed to utilize the idea of variable length trail in the game. Agents are arbitrarily positioned in CyberCromlech nodes at the start of the game. While traversing CyberCromlech, agents form limited length trails $\left(C=C_{0}\right)$. There is also a sweeper-daemon at the end of each agent's trail trying to catch up with the agent. If an agent is trapped, the pursuer catches up by 2 transitions (i.e. trail length is decreased by two: $\mathrm{C}_{\mathrm{t}}=\mathrm{C}_{\mathrm{t}-1}$ - 2). If an agent must cross another's agent trail while moving, the pursuer catches up by one transition (i.e. trail length is decreased by one: $C_{t}=C_{t-1}-1$ ). At the same time the trail of the agent that was just crossed is increased by one (i.e. pursuer's lag interval is increased by one transition: $\left.C_{t}=C_{t-1}+1\right)$. The agent whose pursuer has caught up with it loses. The winner is the agent whose trail is longest after a given number of steps (potential subject to reinterpretation and reformulation).

\subsubsection{Occupancy game}

The game is played by two (or more) teams of agents with an equal number of players. Agents are arbitrarily positioned in the outer level of CyberCromlech nodes at the start of the game. While traversing CyberCromlech, the agents form limited length trail $\left(\mathrm{C}=\mathrm{C}_{0}\right)$. The goal of each team is to maximize the number of nodes "occupied" by trails of its members, nodes where trails of opposing teams cross being disregarded. If an agent is trapped, its trail is decreased by one transition every step it stays put, thereby decreasing the overall teamoccupied territory. The less trapped the team members are, the better the team does overall and vice versa. Therefore, it is a matter of vital importance for one's team to have its agents free and the opposing team's agents trapped. The winning team is announced after a given number of steps. Studying the dynamics of trails' "twining” (e.g. for steady states) is a separate matter posing research interest of its own.

\subsection{Design of game experiments}

The above games can be used to design a number of game experiments for studying the collective behaviour of rational agents. One could assess: 1) the effectiveness of architectures of participating collective agents; 2) advantages and disadvantages of collective behaviour patterns; 3) model-specific human collective behaviour patterns. The main problem in planning the above experiments is that the game models used are exceptionally multifactor which calls for elaboration of the way repeated playoff experiment results are organized and assessed.

\section{TEMPORIZING GAME}

\subsection{Game description}

By nature, the temporizing game resembles a game of timing (Luce and Raiffa 1957), combined with classical maze task. On the first step $\mathrm{N}$ players are arbitrarily positioned in the outer level nodes of CyberCromlech. On every next step each player must make a move, i.e. transition into one of the "open" or "semi-open" adjacent nodes. By passing through the nodes each player forms a trial of "semi-open" and "closed" nodes (trail length unlimited: $\mathrm{C}_{0}=\infty$ ). If a player is trapped at some point, he is out. The player's goal here is to keep moving for as long as possible (i.e. make as many transitions between nodes as possible and not get trapped) and at the same time, to reach the very center of CyberCromlech (the "altar stone") at some point. Reaching the "altar stone" means moving through non"closed" nodes towards the inner area and by entering it, and becoming a contender for victory (Fig.3). At this point the contender for victory is facing the problem of completing it at the right time. 
As soon as any of the players are out, the contender is allowed to move one inner-circle closer to the "altar stone", so that the more players are further out, the closer the contender is to victory. However, if a new contender enters the inner area, all the "old contenders" are out (Fig.3) no matter how close to victory they were (speculation as to how to interpret their current proximity to the center is subject to further interpretation and study). Accordingly, it is the very last contender to enter inner area that gets it all. The game is over when there are no more moving players in CyberCromlech, i.e. when either all the players become contenders for victory or are out. In commemoration of victory the last contender(s) is then allowed to reach the “altar stone” (Fig.4).

\subsection{Game's nature}

The dual character of the game is in the fact that tactically a player must find a way into the maze (formed by other players' trails) and strategically he must carefully choose the moment to become a contender: if he becomes a contender too soon, he risks being kicked out by newer contender(s); on the other hand, staying in the maze for too long, he risks being trapped as the maze structure becomes more complicated on every step. Of specific interest is how these two levels are linked and how they affect collective behaviour patterns under study.

\subsection{Simple algorithm for artificial player}

\subsubsection{Behaviour rules}

To demonstrate basic player logic in the temporizing game let us examine the simple artificial player algorithm. The algorithm is built on simple (possibly, not optimal) implication that if it is ever harder to find a way in the maze at every step, a player is encouraged to become a contender for victory by entering the inner area of CyberCromlech. To denote this factor we introduce the quantitative index of "confidence" (IOC); the higher the IOC is, the less probable it is for the player to leave the maze and enter CyberCromlech inner area and, accordingly, the lower IOC is, the less probable it is for the player to stay in the maze. In such a way, the tactical level of decision making (maze task) is linked to the strategic level (game of timing): if a player follows this rule then the harder it is for him to find his way in the maze, the higher is his motivation to enter the inner area and become a contender.

Assuming the existence of IOC and the fact that a player can only see the adjacent nodes, a player's decision making can take on the following form.

1. Don't go into "closed" nodes.

2. Give priority to "open" nodes over "semi-open" nodes.

3. Give priority to "open" nodes where there are no other players at present.
4. If a number of alternative pathways is less than that of the previous step - decrease the IOC value.

5. The lower the IOC is, the higher the chance to choose among nodes situated closer to the inner area of CyberCromlech.

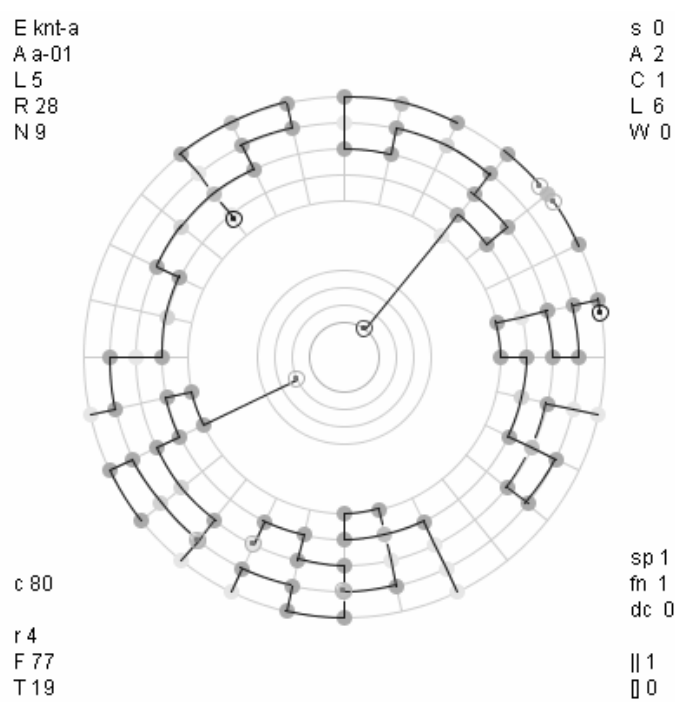

Figure 3. Temporizing game unfolding: "old" contenders are out as new contender arrive

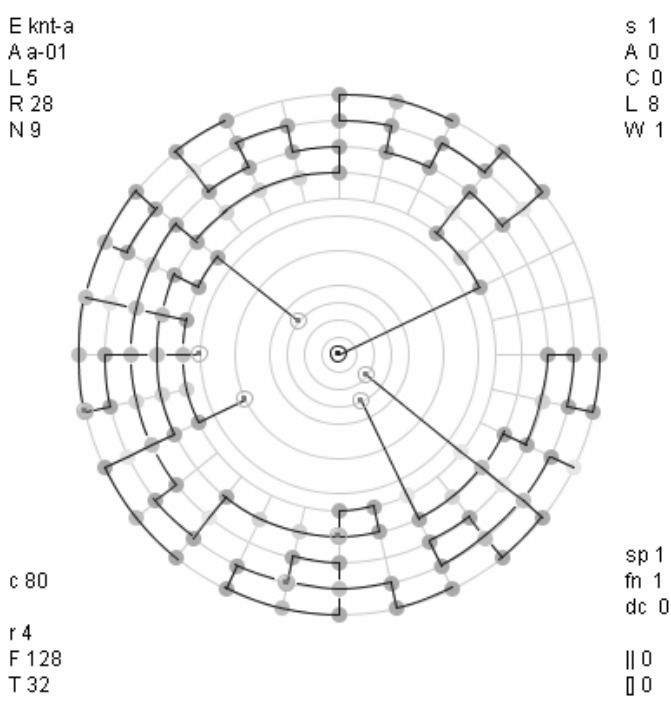

Figure 4. Temporizing game is over with one winner

Two questions must be answered here: firstly, how does one recalculate the node choice probabilities (tactics issue); secondly, how does one change a player's IOC based on available information about the game situation (strategy issue).

\subsubsection{Player's tactics}

The proposed algorithm modifies choice probabilities according to the rules of linear shift of states for graded structure stochastic learning automaton (Tsetlin 1973). 


$$
\begin{aligned}
& p_{i, 1}=p_{i, 0}-\Delta, \\
& p_{j, 1}=p_{j, 0}+\Delta /(n-1), i \neq j,
\end{aligned}
$$

where $\mathrm{p}_{\mathrm{i}}$ - probability of choice for i-th "inconvenient" (according to rules presented above) alternative ( $\mathrm{p}_{\mathrm{i}, 0}-$ before recalculation of probabilities, $\mathrm{p}_{\mathrm{i}, 1}$ - after recalculation), $\mathrm{p}_{\mathrm{j}}$ - probabilities of choice for all other alternatives, $\Delta$ - value, by which probability of choice for i-th "inconvenient" alternative is decreased, $\mathrm{n}-$ current number of alternatives.

\subsubsection{Player's strategy}

Regarding the tuning of the IOC value we propose the following very simple (possibly, not optimal) method:

$$
\begin{aligned}
& \text { if } Q_{i, t}<Q_{i, t-1} \text {, then } c_{i, t}=c_{i, t-1}-\left(Q_{i, t-1}-Q_{i, t}\right) \text {, } \\
& \text { if } Q_{i, t}=Q_{i, t-1} \text { and } Q_{i, t}<3 \text {, then } c_{i, t}=c_{i, t-1}-1 \text {, } \\
& \Delta_{3, i, t}=\Delta_{3, i, t-1}+1 / c_{i, t},
\end{aligned}
$$

where $Q_{\text {i,t-1 }}$ - number of alternatives available to i-th player on previous step of the game, $Q_{i, t}$ - number of alternatives available to i-th player on current step of the game, $c_{i, t}-i$-th player current IOC value, $\Delta_{3, i}-$ value, by which the probability of choice for alternatives, that do not lead a player to a position closer to CyberCromlech's center, is decreased.

That is, according to the above method the IOC value is decreased when there are less convenient alternatives available to a player. Moreover, the IOC value is then decreased by exactly the same number that the number of available alternatives has decreased by. Alternatively, if a player finds himself in the situation where the number of alternatives is less than three and the situation repeats, then the IOC value is decreased by one.

\subsection{Study of the game}

Among possible lines of research (and accordingly computational experiments related to them) the following should be noted.

1. Possibly, the simplest variant is to have repeated playoffs with different types of agent-players where the most effective algorithm of artificial player behaviour (or most effective parameters set for same algorithm) would be determined based on a series of games.

2. Study of the artificial players capable of learning based on their game experience. Here one can plan experimental games, where the learner player will be competing against more "powerful" players (e.g. those more "aware" of the game situation).

3. Of particular importance in context of the above game model is the study of rivalry between "advancedtactics players" and "advanced-strategy players". For that matter, one could specifically imbalance the situation through modifying the player's abilities of solving the maze traversing task (tactics aspect) and abilities of solving the task of becoming a contender at the right time (strategy aspect).

To date a series of computational experiments have been implemented in line with the first line of research with Figure 3 and 4 demonstrating results of temporizing game visualisations. We plan on giving more detail on layout and results of those computational experiments in further publications on the subject.

\section{DISCUSSION}

\subsection{Evolution prospects}

The evolution prospects for the method proposed can be viewed in two aspects: firstly, the complication of the CyberCromlech architecture; secondly, the creation of new games using the CyberCromlech. In the view of the complication of the CyberCromlech architecture, the following variations can be proposed. 1) Increasing the spatial dimensions of CyberCromlech and altering its geometrical configuration (e.g. introducing irregularity in the array of transitions). 2) Altering the rules of transition so that agents could stay in the same node for more than one step (i.e. skipping steps at will). 3) Allowing agents to decide whether to leave the trail while traversing CyberCromlech or not.

\subsection{Connection to other fields of research}

In the view of looking for analogous formalized approaches that could be used to describe and study processes in CyberCromlech, the following could be considered: 1) random walk studies related to the Monte-Carlo method, 2) studies in the field of graphical interpretation of words and languages, including "turtle interpretation" of words; 3) the mathematical theory of knots.

\subsection{Applying the approach}

CyberCromlech can be effectively used both for multipurpose modelling of multiagent interactions and construction of production multiagent systems. Detailed examination of such applications is the subject of further publications on the matter. Meanwhile, we would like to draw you attention to the following.

1. Multi-player computer game development. It is implied here that a human player gets to control one or several agents traversing the CyberCromlech. It must be specifically noted that spontaneous emergence and evolution of trails formed by agents could be used to create games with non-linear plots, individual for each player.

2. Design of routing algorithms for overlay in peer-topeer computer networks. In this case peers can be considered as nodes of CyberCromlech, whereas data packets play agents traversing it. The trail formed by a 
particular agent can be regarded as a route connecting the network nodes which the respective data packet passed on its way.

3. Design of systems of story generation. CyberCromlech agents could, for example, play the role of story characters whilst the game space would present them with possible action alternatives but leaving final decisions as to where to go to their discretion. Accordingly, the game setup would be deemed equivalent to the story's basic idea whilst agents' traversals would be regarded as separate interrelated story lines.

\section{CONCLUSIONS}

A new approach to the design of game experiments (test problems) based on CyberCromlech is proposed in the paper. The approach is based on the idea of storing and utilizing the history of agent interactions in a form explicit and convenient enough to allow for the study of the spontaneous emergence of collective behaviour. A number of games have been examined to demonstrate the capabilities of CyberCromlech in providing for that matter. Apart from this, the most elaborate of them - the temporizing game - is examined in more detail using the proposed simple algorithm of artificial player behaviour. In conclusion, the vision of further evolution and potential practical application of the proposed approach is given.

\section{REFERENCES}

Botchkaryov A. 2002. Solving the problem of mechanical balancing by collective of mobile agents, Transactions on Computer systems and networks, Lviv Polytechnic National University Press, No. 463, pp.14-18 (Ukr.)

Ishida T. 1997. Real-Time Search for Learning Autonomous Agents, Kluwer Academic Publishers.
Pollack M. and Ringuette M. 1990. Intoducting the Tileworld: Experimentally Evaluating Agen Architectures, Proceedings of the $8^{\text {th }}$ National Conference on Artificial Intelligence (AAAI-90), Boston, MA, pp. 183-189.

Luce D. and Raiffa H. 1957. Games and decisions, Intoduction and critical survey, London, Chapman and Hall.

Tsetlin M. 1973. Automaton Theory and Modeling of Biological Systems, Academic Press, New York.

Weiss G. (Editor) 2000. Multiagent Systems: A Modern Approach to Distributed Artificial Intelligence, MIT Press.

Woolridge M. 2002. An Introduction to MultiAgent Systems, John Wiley \& Sons.

\section{AUTHOR BIOGRAPHIES}

ALEXEY BOTCHKARYOV earned his master's degree in Computer and Intelligent Systems and Networks from Lviv Polytechnic National University (Ukraine, Lviv) in 1998. From September 2001 to date he has been holding senior lecturer position at Lviv Polytechnic National University. Alexey's main research interests include development of multiagent systems and development of intelligent distributed exploration systems based on multiagent systems' technology.

DR. SERHIY KOVELA earned his master's and PhD degrees in Computer Science from the Lviv Polytechnic National University (Ukraine, Lviv) in 1996 and 2000, respectively. From 1996 till August 2003 he held senior lecturer position at Lviv Polytechnic and since September 2003 to date - senior lecturer position at Kingston University, London. Serhiy's main research interests include automation of design of business information systems and design of Web-enabled middleware systems. 